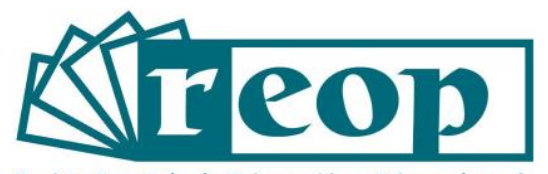

Revista Española de Orientación y Psicopedagogía

\title{
EVALUACIÓN DE CONDUCTAS Y ACTITUDES DE BÚSQUEDA DE EMPLEO. REVISIÓN Y VALIDACIÓN DE TRES ESCALAS PARA LA ORIENTACIÓN PROFESIONAL
}

\section{ASSESSMENT OF JOB SEARCH BEHAVIORS AND ATTITUDES. REVIEW AND VALIDATION OF THREE SCALES FOR CAREER GUIDANCE}

\author{
Rafael Piqueras Gómez ${ }^{1}$ \\ Universidad de Murcia. Facultad de Ciencias del Trabajo. Máster Interuniversitario en Orientación \\ e Intermediación Laboral. Murcia, España \\ Antonio Carrasco Hernández \\ Universidad de Murcia. Facultad de Ciencias del Trabajo. Departamento de Organización de \\ Empresas y Finanzas. Murcia, España \\ José Luis Rodríguez-Arias Palomo \\ Servicio Gallego de Salud. A Coruña, España \\ Ángel Altuna Urcelay \\ Lanbide-Servicio Vasco de Empleo. Vitoria-Gasteiz. España
}

\section{RESUMEN}

Uno de los objetivos fundamentales de la orientación profesional es facilitar la integración laboral. Para ello, necesita contar con instrumentos válidos y fiables que evalúen el proceso de búsqueda de empleo. Se pretende ampliar y mejorar tres escalas utilizadas por el Servicio de Empleo y Formación de la Región de Murcia (SEF) dirigidas a medir los principales elementos cognitivoconductuales implicados en el proceso de búsqueda de empleo: sistema facilitador de inserción (SFI), conductas de búsqueda de empleo (ICBE) y expectativas de búsqueda de empleo

${ }^{1}$ Correspondencia: Rafael Piqueras Gómez. Correo-e: rafaelpiqueras@gmail.com 
(ECPBE). Después de estudiar su fiabilidad y validez se eliminan algunos ítems y se añaden nuevos elementos basados en la revisión de la literatura y la opinión de expertos. Las escalas mejoradas se someten a validación mediante análisis factorial confirmatorio de una muestra de 472 personas. Atendiendo a los resultados, las nuevas escalas presentan una fiabilidad adecuada en todos sus elementos con un alfa de Cronbach superior a .7. Muestran una adecuada estructura $y$ ajuste en el modelo de medida robusto Satorra-Bentler $x 2(494)=972.43(p=.000), N F I=.9$, $\mathrm{NNFI}=.92, \mathrm{CFI}=.93$ y $\mathrm{RMSEA}=.05$ con una correcta validez convergente y discriminante. Se concluye que, las escalas mejoradas constituyen una medida válida y fiable de las principales variables implicadas en la búsqueda de empleo y una herramienta útil para la toma de decisiones basadas en la evidencia sobre la idoneidad de las actuaciones de orientación profesional dirigidas a personas desempleadas.

Palabras clave: orientación profesional, búsqueda de empleo, expectativas, proactividad, análisis factorial confirmatorio, integración laboral.

\section{ABSTRACT}

One of the fundamental objectives of career guidance is to facilitate labor integration. To do this you need to have valid and reliable instruments that evaluate the job search process. It is intended to expand and improve three scales used by the Employment and Training Service of the Region of Murcia (SEF) aimed at measuring the main cognitive-behavioral elements involved in the job search process: insertion facilitator system (SFI), search behaviors employment (ICBE) and job search expectations (ECPBE). After a preliminary study, some items are removed, and new elements are added based on the literature review and expert opinion. The improved scales are subject to validation by confirmatory factor analysis on a sample of 472 people. According to the results, the new scales have adequate reliability in all their elements with a Cronbach's alpha greater than .7. They show an adequate structure and fit in the robust Satorra-Bentler measurement model $\mathrm{X} 2(494)=972.43(\mathrm{p}=.000), \mathrm{NFI}=.9, \mathrm{NNFI}=.92, \mathrm{CFI}=.93$ and RMSEA $=$ .05 with a correct convergent and discriminant validity. In conclusion, the improved scales constitute a valid and reliable measure of the main variables involved in the job search and a useful tool for decision-making based on evidence about the suitability of career guidance actions aimed at unemployed people.

Key Words: career guidance, job search, expectancy, proactivity, confirmatory factor analysis, labor integration.

\section{Cómo citar este artículo:}

Piqueras, R., Carrasco, A., Rodríguez-Arias, J.L. y Altuna, Á. (2021). Evaluación de conductas y actitudes de búsqueda de empleo. Revisión y validación de tres escalas para la orientación profesional. Revista Española de Orientación y Psicopedagogía, 32(2), 69-90. https://doi.org/10.5944/reop.vol.32.num.2.2021.31279 


\section{Introducción}

El Consejo de la Unión Europea (2008) define la orientación permanente como:

“...el proceso continuo que permite a los ciudadanos, a cualquier edad y a lo largo de su vida, determinar sus capacidades, sus competencias y sus intereses, tomar decisiones en materia de educación, formación y empleo y gestionar el recorrido de su vida personal en lo que respecta a la educación y la formación, en el trabajo y en otros marcos en los que es posible adquirir o utilizar estas capacidades y competencias" (p. 1).

Con el objetivo de facilitar este proceso, los servicios educativos y de empleo atienden a miles de ciudadanos que desean desarrollarse social y laboralmente de manera positiva. Cuando el motivo primordial de estas personas es la consecución de empleo, cuentan con la ayuda de profesionales capacitados para facilitar su proceso de inserción. Estos técnicos se conocen como orientadores profesionales y utilizan distintas estrategias de intervención que requieren de instrumentos de medida específicos para poder valorar tanto a las personas que buscan trabajo como el impacto de las actuaciones aplicadas durante el proceso de inserción.

Las primeras intervenciones específicas de ayuda a la búsqueda de empleo son de carácter conductual: inciden principalmente en el entrenamiento en habilidades de búsqueda mediante programas estructurados de modificación de conducta. Su referente más conocido son los denominados Job-clubs (Azrin et al., 1975). Con el tiempo se añaden otros enfoques que muestran más eficacia, destacando en este sentido los modelos cognitivos (Aramburu, 2003). La perspectiva cognitiva se concreta otorgando más relevancia a aspectos como las expectativas de autoeficacia (Mortimer et al., 2016; Rego-Agraso y Rial-Sánchez, 2018; Saks et al., 2015), el locus de control (Caliendo et al., 2015; Preuss y Hennecke, 2018; Trice et al., 1989), el trabajo con los objetivos (Karsten et al., 2016; Tolentino et al., 2014) o la actitud proactiva (Pan et al., 2018; Salessi y Omar, 2018; Seibert et al., 1999).

Con el tiempo se impone un modo de intervención que integra elementos motivacionales y conductuales como mejor forma de aumentar las probabilidades de inserción (Nieto Flores et al., 2015). En este sentido, el metaanálisis realizado por Liu et al. (2014), revela que los programas que incluyen conjuntamente el desarrollo de habilidades, la mejora de la autoestima, el trabajo con los objetivos de búsqueda y la proactividad, aumentan más las probabilidades de inserción que si sólo se aplica uno de los enfoques.

En España, los servicios de empleo incluyen estos elementos en los manuales y guías que utiliza su personal técnico (INEM, 2000푸 Pablo, 1996; SEF, 2007; SENAE, 2010; Teré, 2019), intervenciones que se caracterizan por otorgar al orientador profesional un papel de agente activo que facilita la inserción desde los propios recursos de la persona que busca trabajo y que procura generar expectativas positivas que mejoren sus probabilidades de inserción (Palenzuela, 1989; Sorić et al., 2019). Estas guías y manuales cuentan con instrumentos de medida como encuestas de satisfacción, fichas de seguimiento o escalas de autovaloración; herramientas útiles para ayudar a los profesionales en la toma de decisiones, pero que en contadas ocasiones han sido convenientemente validadas para su uso.

Existen estudios que utilizan diversas escalas de medida relacionadas con elementos cognitivo-conductuales vinculados a la situación de búsqueda de empleo (Cifre et al., 2018; Hernández-Fernaud et al., 2011; Nieto-Flores et al., 2019). Sin embargo, suelen ser trabajos puntuales provenientes del ámbito académico que no llegan a incorporarse al quehacer diario de los servicios de orientación profesional para el empleo. 


\section{Fundamentación teórica}

En 2009, el Servicio Público de Empleo de la Región de Murcia (SEF) crea una Plataforma de Orientación Laboral (POL) como herramienta informática de apoyo a la orientación que incluye las tres escalas psicométricas objeto de este estudio. La construcción de las escalas se basa en tres tópicos fundamentales: la Teoría General de los Sistemas (Von Bertalanffy, 1993), el construccionismo social (McNamee y Gergen, 1996) y la teoría de la comunicación humana (Watzlawick et al., 1981).

Desde este enfoque, las escalas evalúan la situación de desempleo como un sistema abierto en el que interactúan diferentes elementos para alcanzar el objetivo del empleo y lo hacen bajo los axiomas de la comunicación, interactuando con el orientador profesional y otras personas relevantes para construir un relato centrado en los recursos de la persona que confíe en su iniciativa y aumente las posibilidades de inserción laboral (O'Hanlon y Weiner-Davis, 1990; Piqueras y Rodríguez, 1997). En este marco teórico, cobran especial importancia las expectativas de control y los elementos comportamentales de proactividad y búsqueda activa de empleo.

\section{Objetivo}

El objetivo de este trabajo es revisar la fiabilidad y validez de tres de las escalas utilizadas por el SEF y proponer una nueva versión, sometida también a análisis, que constituya una propuesta más completa para ser utilizada por técnicos, profesionales de la orientación e investigadores en este campo.

\section{Procedimiento}

Se revisan los estudios anteriores sobre la validez de las escalas originales (Piqueras et al., 2016) y se completa su conocimiento con un análisis exploratorio (Hefetz y Liberman, 2017) de datos de usuarios de orientación del SEF. A partir de este análisis y de los nuevos elementos aportados por los expertos, se construyen las escalas mejoradas y se aplican a una muestra de personas desempleadas. Se usa el modelo de medición mediante análisis factorial confirmatorio (AFC), tanto de los ítems como de las dimensiones de orden superior en las que se organizan jerárquicamente. Se aplica el modelo de ecuaciones estructurales para mínimos cuadrados parciales (PLS-SEM) ya que no se cumple el supuesto de normalidad multivariante (Chou et al., 1991; West et al., 1995). Para un análisis más completo, también se aplica el modelo de ecuaciones estructurales basado en las estructuras de covarianza (CB-SEM) (Hair et al., 2010) con las correcciones aplicadas por Bentler (Bentler, 2006). Se realiza el análisis de validez convergente y discriminante y el análisis de los resultados predictivos de los modelos. Para el análisis de los datos se utiliza SPSS 25. EQS 6.4 y SMARTPLS 3.2.8.

\section{Instrumentos}

Las principales características de las escalas originales incluidas en el estudio son: 
- Sistema facilitador de inserción (SFI). Su función es valorar de manera sencilla los cuatro elementos del sistema facilitador de inserción - información, planificación, habilidades y motivación - propuestos por los expertos orientadores participantes en el desarrollo de las guías técnicas del Instituto Nacional de Empleo (INEM, 2000b; Montilla, 2003; SENAE, 2010). La escala consta de 6 ítems que valoran el nivel de acuerdo en formato tipo Likert de 1 a 9 puntos. Tal y como se observa en la Tabla 1, incluye tres elementos dirigidos a los aspectos más conductuales relativos a la información, la planificación y las habilidades de búsqueda y dos para valorar la motivación. Se añade un sexto elemento como medida del valor del trabajo por su relevancia en los estudios de psicología social del trabajo (Blanch, 2006; Agulló, 1998).

- Inventario de conductas de búsqueda de empleo (ICBE). Construido por el personal técnico experto en orientación del SEF como medida objetiva de la variedad y organización de las acciones de búsqueda. En la Tabla 1 se observa que incluye dos partes: una primera que mide el factor técnicas de búsqueda, en la que se debe señalar cuáles realiza y con qué frecuencia (ocasional/mensual/semanal/diaria) y una segunda que valora el factor organización, señalando la forma de planificación (Nunca/A veces/Bastante/Siempre) y un indicador de intensidad según las horas semanales dedicadas a buscar trabajo (0-5/ 5-10 / 10-20 / más de 20).

- Expectativas de control percibido de búsqueda de empleo (ECPBE). Es la escala más antigua de las tres y ha demostrado una adecuada fiabilidad y validez (Piqueras et al., 2016). Forma parte de varias guías de orientación publicadas en España y Latinoamérica (INEM, 2000a; SENAE, 2010) y de estudios empíricos (Montilla, 2005; Pérez-Vacas, 2016). Tal y como se observa en las Tablas 1 y 2, la escala incluye 12 ítems tipo Likert de 1 a 9 puntos que se dividen a su vez en cuatro subescalas con tres ítems cada una: autoeficacia, éxito, locus de control interno y locus de control externo.

Tabla 1

Escalas originales

\section{Sistema facilitador de inserción (SFI)}

\begin{tabular}{lc}
\hline Ítem & Indicador \\
\hline -Tengo toda la información que necesito para conseguir trabajo. & \\
-Me organizo habitualmente la búsqueda de empleo. & \\
-Domino las técnicas de búsqueda de empleo (currículum, anuncios, entrevistas, & Comportamientos \\
Internet, etc.). & \\
\hline -Me siento muy animada/o para buscar trabajo. & Motivación \\
\hline -Creo que voy a conseguir pronto empleo. & Valor del trabajo \\
\hline -Lo más importante en la vida es trabajar. &
\end{tabular}

Fuente: SENAE (2010) y elaboración propia 


\section{Tabla 2}

Escalas originales (Continuación)

Inventario de conductas de búsqueda de empleo (ICBE)

\begin{tabular}{lc}
\hline Ítem & Indicador \\
\hline -Pregunto a familiares o conocidos sobre empleo. & Técnicas de \\
-Consulto anuncios de prensa. & búsqueda \\
-Miro ofertas en tablones de anuncios. & -Visito oficinas de empleo o ETTs. \\
-Acudo a las instituciones y organismos públicos. & Organización \\
-Me presento a empresas privadas. & Intensidad \\
\hline
\end{tabular}

\section{Expectativas de control percibido de búsqueda de empleo (ECPBE)}

\begin{tabular}{ll}
\hline Ítem & Indicador \\
\hline
\end{tabular}

-Soy capaz de soportar los inconvenientes que conlleva la búsqueda de empleo.

-Estoy capacitada/o para superar los rechazos cuando busco empleo.

Autoeficacia

-Creo que puedo comportarme adecuadamente en una entrevista de trabajo.

-Creo que estaré poco tiempo en el paro.

-Tengo muchas esperanzas de que me den trabajo.

-Creo que hay muchas posibilidades de que termine encontrando un buen empleo.

-Cuanto más me esfuerce en buscar trabajo más posibilidades tendré de encontrarlo.

-Encontrar trabajo es algo que va a depender, fundamentalmente, de mí.

-Encontrar trabajo dependerá del tiempo que dedique a buscarlo.

-Para encontrar trabajo tendré que esperar a que mejore mi suerte.

-Lo que más me ayudaría a encontrar trabajo es contar con parientes y conocidos influyentes.

-Sin recomendaciones es muy difícil conseguir trabajo.

Fuente: SENAE (2010) y elaboración propia

\section{Revisión de las escalas originales}

Existen estudios previos que ya muestran buenos resultados sobre la capacidad de las escalas originales para prevenir la duración del desempleo (Piqueras et al., 2008) o para valorar el impacto del paro de larga duración en personas mayores de 45 (Piqueras et al., 2018; Sousa-Ribeiro et al., 2018). Para estudiar con mayor profundidad su comportamiento estadístico, se extraen los datos de 25.814 personas desempleadas que inician una intervención de orientación en el SEF y cumplimentan las escalas entre los años 2010 y 2014. Se comprueba que la fiabilidad de las tres escalas obtiene un alfa de Cronbach mayor de .7 en todos sus elementos, excepto la subescala 
de locus de control externo del ECPBE que tan sólo alcanza el .55. También se realiza un análisis factorial exploratorio (Hair et al., 2019) de las escalas originales donde se observa que el ítem del SFI referido al valor del trabajo no se agrupa con el resto de los ítems de su escala.

Con los datos obtenidos en la revisión se considera justificada la eliminación de algunos elementos y la incorporación de nuevos ítems acordes con los planteamientos teóricos. En concreto se revisa la homogeneidad y coherencia de los ítems con el fin de eliminar los que no responden a los presupuestos en los que se basan las escalas y se prescinde de los ítems que no superan una fiabilidad de .7. También se completan algunos aspectos que el análisis factorial confirmatorio saca a la luz constituyendo nuevos constructos de orden superior que mejoran la definición de las subescalas. Así mismo, se construyen nuevos ítems para la evaluación de la proactividad como elemento fundamental a la hora de predecir la inserción laboral por las evidencias científicas (Liu et al., 2014).

\section{Construcción de las escalas mejoradas}

A partir del análisis de los datos originales, se realizan las siguientes modificaciones: del SFI se elimina el ítem referido al valor del trabajo ya que no se agrupa con el resto de los elementos de su escala al responder a un factor teórico más relacionado con la psicología social que con el enfoque centrado en los recursos que inspiró la configuración del sistema facilitador de inserción (INEM, 2000b).

En el ICBE, se añaden dos ítems para diferenciar mejor los distintos tipos de técnicas de búsqueda de empleo: informativas (dirigidas a conseguir información sobre ofertas de trabajo), de contacto personal (técnicas de autopresentación que implican la consecución de entrevistas con personas de empresas o instituciones relacionadas con el empleo) y de contacto diferido (uso de herramientas o servicios intermedios para buscar trabajo).

Respecto al ECPBE, se sustituye su denominación por la de Conductas Motivadas de Búsqueda de Empleo (COMOBE) para describir mejor su contenido ya que se elimina la subescala de locus de control externo - por su bajo índice de fiabilidad - y se incluyen nuevos elementos referidos al comportamiento proactivo (Salessi y Omar, 2017). Así, el COMOBE mantiene la medida de las tres expectativas positivas que configuran el constructo de optimismo fundado: autoeficacia, éxito y locus de control interno (Contador et al., 2012; Palenzuela, 1989) y añade 11 nuevos ítems de proactividad agrupados en tres elementos: divergencia (4 ítems relacionados con la búsqueda de alternativas y nuevas formas de afrontar la búsqueda), iniciativa (4 ítems sobre el proceso auto-motivado y no coaccionado de actuación) y futuro (3 ítems sobre la orientación a producir cambios para obtener objetivos), propuestos por los autores de este estudio de acuerdo con los criterios identificativos de la conducta proactiva (Salessi y Omar, 2017). Las escalas mejoradas se pueden ver en el Apéndice.

\section{Muestra de las escalas mejoradas}

Con el fin de realizar una prospección lo más amplia y representativa posible para el estudio de las escalas mejoradas, se aplican los cuestionarios a 472 personas desempleadas durante los meses de diciembre de 2018 y enero de 2019. Realizan la recogida de datos técnicos del SEF pertenecientes a 5 oficinas de empleo, al servicio de formación y a dos entidades colaboradoras. Finalmente se recogen cuestionarios válidos de 271 mujeres $(57.42 \%)$ y 201 hombres $(42.58 \%)$. La edad media de los encuestados es de 40.39 años con una desviación típica de 9.8 y un error 
muestral del $4.51 \%$ para un $\mathrm{p}=\mathrm{q}=.5$ y un nivel de confianza del .95. La muestra presenta una distribución por sexo similar a la publicada por el Instituto Nacional de Estadística en el primer trimestre de 2019, tanto para la Región de Murcia (57.08\% mujeres y $42.92 \%$ hombres) como para España (53.18\% mujeres y $46.82 \%$ hombres).

\section{Resultados}

Con los datos recogidos en las escalas mejoradas, se realiza un análisis de patrones sospechosos de respuesta, el sesgo de método común (MacKenzie y Podsakoff, 2012), el factor latente común (Gaskin y Happell, 2014), los casos perdidos (Hair et al., 2019) y el sesgo de no respuesta (Hair et al., 2013). No se encuentran resultados que indiquen problemas estadísticos en los datos. Se examina la asimetría y curtosis de las variables con las pruebas de Kolmogorov-Smirnov y Shapiro-Wilks y se aplica la prueba de Mardia (1970) de curtosis multivariante (normalizada $37.8 \mathrm{p}$ $<.001$ ), que muestra que el supuesto de normalidad multivariante no se cumple. Del análisis de fiabilidad con los datos de las escalas mejoradas se comprueba que todos los elementos se agrupan con un alfa de Cronbach superior a .7.

Bondad de ajuste y análisis del modelo de medida de las escalas mejoradas

En PLS-SEM, el principal indicador de ajuste utilizado es SRMR (standardized root mean square residual), que ofrece un buen ajuste con un valor de .04. Los estadísticos inferenciales Bootstrap (SRMR y dULS) en el modelo estimado son, en el caso de SRMR, .08 y de dULS, 5.62, dentro del intervalo basado en Bootstrap del 95\%, lo que muestra un buen ajuste. En CB-SEM, se estiman los indicadores de ajuste recomendados (Anderson y Gerbing, 1988; Fornell y Larcker, 1981a). Se obtiene un buen ajuste de medida robusto: Satorra-Bentler $X 2(636)=681.87(p=.10)$ y de los indicadores: $[\mathrm{NFI}]=.9,[\mathrm{NNFI}]=.93,[\mathrm{CFI}]=.93$ y $[\mathrm{RMSEA}]=.06$

\section{Análisis del modelo de medida}

Fiabilidad individual del ítem: Se examinan las relaciones de cada ítem con su respectivo constructo. En las Tablas 3 y 4 se observa que todos los indicadores individuales para cada ítem, tanto en CB-SEM como en PLSE-SEM, presentan valores significativos del coeficiente estandarizado a un nivel del $95 \%(p<.05)$ con una carga $(\lambda)$ superior a .7 .

Fiabilidad o consistencia interna: Se evalúa si las variables observables están midiendo rigurosamente a la variable latente a la que representan. En las tablas 3 y 4 , se comprueba que las escalas presentan un valor adecuado de fiabilidad compuesta ( $\rho$ c), al ser superiores 0 próximos a .8, (Bagozzi y Yi, 1988 y 2012; Fornell y Larcker, 1981a) y nunca superiores a .95 (Hair et al., 2019). 
Validez convergente: Valora si un conjunto de indicadores mide realmente un constructo determinado y no están midiendo otro distinto. Se utiliza la varianza extraída media (AVE) y, tal y como se observa en las Tablas 3 y 4 , ofrece un valor superior al límite recomendable de .5 (Bentler, 2006; Hair et al., 2019).

\section{Tabla 3}

Resultados del modelo de las escalas mejoradas

\begin{tabular}{|c|c|c|c|c|c|}
\hline Ítems* & $\begin{array}{c}\text { Cargas } \\
(\lambda) \\
\text { CB-SEM }\end{array}$ & $\begin{array}{c}\text { Cargas } \\
(\lambda) \\
\text { PLS- } \\
\text { SEM }\end{array}$ & $\begin{array}{c}\text { Intervalo } \\
\mathbf{R}^{2}\end{array}$ & $\begin{array}{c}\text { Fiabilidad } \\
\text { compuesta } \\
\text { (pc) } \\
{[5 \%-95 \%]}\end{array}$ & $\begin{array}{c}\text { AVE } \\
{[5 \%-95 \%]}\end{array}$ \\
\hline \multicolumn{6}{|c|}{ 1- SFI/Comportamientos } \\
\hline $\mathrm{CO} 1$ & .73 & .73 & $.53-.53$ & .839 & .636 \\
\hline $\mathrm{CO} 2$ & .89 & .89 & $.79-.79$ & {$[.808-.865]$} & {$[.586-.68$} \\
\hline $\mathrm{CO} 3$ & .78 & .77 & $.59-.61$ & & \\
\hline
\end{tabular}

2- SFI/Motivación

\begin{tabular}{lccccc} 
MO4 & .85 & .79 & $.59-.62$ & .789 & .651 \\
MO5 & .77 & .82 & $.67-.72$ & {$[.752-.821]$} & {$[.603-.696]$} \\
\hline 3- ICBE/FRECUENCIA/Información & & \\
IF1 & .7 & .73 & $.49-.53$ & .836 & .630 \\
IF2 & .76 & .78 & $.58-.61$ & {$[.810-.859]$} & {$[.588-.671]$} \\
IF3 & .9 & .87 & $.76-.81$ & &
\end{tabular}

4- ICBE/FRECUENCIA/Gestión personal

$\begin{array}{llllcc}\text { GP4 } & .74 & .85 & .55-.72 & .862 & .610 \\ \text { GP7 } & .78 & .74 & .55-.61 & {[.837-.882]} & {[.564-.651]} \\ \text { GP8 } & .76 & .79 & .58-.62 & & \\ \text { GP10 } & .74 & .74 & .55-.55 & & \end{array}$

5- ICBE/FRECUENCIA/Gestión diferida

$\begin{array}{lccccc}\text { GD5 } & .7 & .74 & .49-.55 & .807 & .582 \\ \text { GD6 } & .83 & .8 & .64-.69 & {[.775-.835]} & {[.536-.628]} \\ \text { GD9 } & .77 & .75 & .56-.59 & & \end{array}$

6- ICBE/Organización

\begin{tabular}{lccccc} 
OR11 & .78 & .71 & $.50-.61$ & .830 & .550 \\
OR12 & .85 & .77 & $.59-.72$ & {$[.806-.851]$} & {$[.510-.589]$} \\
OR13 & .79 & .77 & $.59-.62$ & & \\
OR14 & .7 & .72 & $.49-.52$ & & \\
\hline
\end{tabular}

7- COMOBE/OPTIMISMO FUNDADO/Autoeficacia

$\begin{array}{lccccc}\text { AU2 } & .81 & .83 & .66-.69 & .820 & .604 \\ \text { AU8 } & .8 & .79 & .62-.64 & {[.787-.0849]} & {[.553-.654]} \\ \text { AU14 } & .74 & .7 & .49-.55 & & \end{array}$

* Los ítems se identifican por dos letras del indicador y el nº de orden en su escala. (Para consultar el contenido de los ítems, ver el Apéndice.)

Fuente: Elaboración propia 


\section{Tabla 4}

Resultados del modelo de las escalas mejoradas (Continuación)

\begin{tabular}{|c|c|c|c|c|c|}
\hline Ítems* & $\begin{array}{c}\text { Cargas } \\
(\lambda) \\
\text { CB-SEM }\end{array}$ & $\begin{array}{l}\text { Cargas } \\
(\lambda) \\
\text { PLS- } \\
\text { SEM }\end{array}$ & $\begin{array}{c}\text { Intervalo } \\
\mathbf{R}^{2}\end{array}$ & $\begin{array}{c}\text { Fiabilidad } \\
\text { compuesta } \\
\text { (pc) } \\
\text { [5\%-95\%] }\end{array}$ & $\begin{array}{c}\text { AVE } \\
\text { [5 \%-95 \%] }\end{array}$ \\
\hline \multicolumn{6}{|c|}{ 8- COMOBE/OPTIMISMO FUNDADO/Éxito } \\
\hline EX4 & .81 & .76 & $.58-.66$ & .860 & .672 \\
\hline EX10 & .82 & .8 & $.64-.67$ & {$[.834-.882]$} & {$[.627-.714]$} \\
\hline EX16 & .83 & .9 & $.69-.81$ & & \\
\hline \multicolumn{6}{|c|}{ 9- СОМОВE/OPTIMISMO FUNDADO/Locus interno } \\
\hline LI6 & .83 & .79 & $.62-.69$ & .808 & .585 \\
\hline LI12 & .76 & .78 & $.58-.61$ & {$[.776-.838]$} & {$[.537-.633]$} \\
\hline LI18 & .7 & .72 & $.49-.52$ & & \\
\hline \multicolumn{6}{|c|}{ 10- СOMOBE/PROACTIVIDAD/Divergencia } \\
\hline DI11 & .71 & .72 & $.50-.52$ & .854 & .596 \\
\hline DI15 & .77 & .73 & $.53-.59$ & {$[.831-.875]$} & {$[.553-.638]$} \\
\hline DI17 & .83 & .83 & $.69-.69$ & & \\
\hline DI19 & .77 & .8 & $.59-.64$ & & \\
\hline \multicolumn{6}{|c|}{ 11- COMOBE/PROACTIVIDAD/Iniciativa } \\
\hline IN3 & .73 & .7 & $.49-.53$ & .828 & .547 \\
\hline IN7 & .73 & .77 & $.53-.59$ & {$[.801-.851]$} & {$[.503-.589]$} \\
\hline IN13 & .74 & .78 & $.55-.61$ & & \\
\hline IN20 & .71 & .71 & $.50-.50$ & & \\
\hline \multicolumn{6}{|c|}{ 12- СOMOBE/PROACTIVIDAD/Futuro } \\
\hline FU1 & .77 & .77 & $.59-.59$ & .793 & .563 \\
\hline FU5 & .7 & .72 & $.49-.52$ & {$[.758-.825]$} & {$[.512-.612]$} \\
\hline FU9 & .81 & .76 & $.58-.65$ & & \\
\hline
\end{tabular}

* Los ítems se identifican por dos letras del indicador y el no de orden en su escala. (Para consultar el contenido de los ítems, ver el Apéndice.)

Fuente: Elaboración propia

Validez discriminante: Comprueba si un constructo determinado mide un concepto distinto a otros constructos. Para ello, tiene que cumplirse que la varianza que un constructo comparta con sus indicadores sea mayor que la que pueda compartir con otros constructos incluidos en el modelo; por tanto, la varianza extraída media (AVE) de cada constructo debe ser mayor al cuadrado de las correlaciones entre el constructo y otras variables latentes del modelo (Fornell y Larcker, 1981b).

Como puede verse en la tabla 5, la raíz cuadrada del AVE para todas las construcciones es mayor que la correlación entre ellas. El análisis de correlación revela una fuerte asociación entre las dimensiones que integran cada escala, especialmente en COMOBE. El componente motivación del SFI, presenta una fuerte asociación con la dimensión iniciativa del COMOBE. 


\section{Tabla 5}

Validez discriminante

\begin{tabular}{lcccccccccccc}
\hline Componentes & $\mathbf{1}$ & $\mathbf{2}$ & $\mathbf{3}$ & $\mathbf{4}$ & $\mathbf{5}$ & $\mathbf{6}$ & $\mathbf{7}$ & $\mathbf{8}$ & $\mathbf{9}$ & $\mathbf{1 0}$ & $\mathbf{1 1}$ & $\mathbf{1 2}$ \\
\hline 1-Comportamientos &. $\mathbf{8}$ & & & & & & & & & & & \\
2-Motivación & .55 & $\mathbf{. 8 1}$ & & & & & & & & & & \\
3-Información & .35 & .3 &. $\mathbf{7 9}$ & & & & & & & & & \\
4-Gestión personal & .39 & .25 & .56 & .78 & & & & & & & & \\
5-Gestión diferida & .47 & .24 & .52 & .6 & .76 & & & & & & & \\
6-Organización & .56 & .4 & .59 & .65 & .62 & .74 & & & & & & \\
7-Autoeficacia & .47 & .44 & .23 & .31 & .38 & .38 & .78 & & & & & \\
8-Éxito & .52 & .58 & .38 & .46 & .47 & .5 & .72 & .74 & & & & \\
9-Locus interno & .35 & .48 & .3 & .28 & .32 & .34 & .7 & .67 & .77 & & & \\
10-Divergencia & .5 & .51 & .33 & .32 & .41 & .42 & .75 & .72 & .67 & .77 & & \\
11-Iniciativa & .3 & .79 & .17 & .21 & .21 & .27 & .51 & .59 & .61 & .55 & .82 & \\
12-Futuro & .55 & .5 & .23 & .31 & .39 & .41 & .74 & .72 & .65 & .69 & .51 & .75 \\
\hline
\end{tabular}

Fuente: elaboración propia

También se comprueba la relación heterotrait-monotrait (HTMT): Las correlaciones heterotrait (entre ítems de distintos constructos) son más pequeñas que las correlaciones monotrait (entre ítems del mismo constructo), tal y como recomiendan Henseler et al. (2015), lo que indica que el modelo es adecuado para medir los constructos especificados (Gold et al., 2001). En la Figura 1, se puede observar el modelo del análisis factorial confirmatorio (AFC) para las escalas mejoradas con todos los ítems que pueden consultarse en el Apéndice.

\section{Modelos de orden superior}

Los modelos de orden superior recogen constructos de segundo orden o superiores y ayudan a medir fenómenos complejos y más abstractos. Para ello, se desarrollan modelos de ecuaciones estructurales de orden superior según las recomendaciones de Koufteros et al. (2009). El SFI se identifica con dos dimensiones de primer orden, el ICBE con seis dimensiones de primer orden y el COMOBE con seis dimensiones de primer orden. Siguiendo las recomendaciones de Rindskopf y Rose (1988), todas las dimensiones tienen más de dos ítems, excepto la dimensión motivación del SFI que se ha estimado con sólo dos ítems, tal y como se puede apreciar en el Apéndice. 


\section{Figura 1}

AFC del modelo para las escalas mejoradas SFI, ICBE y COMOBE

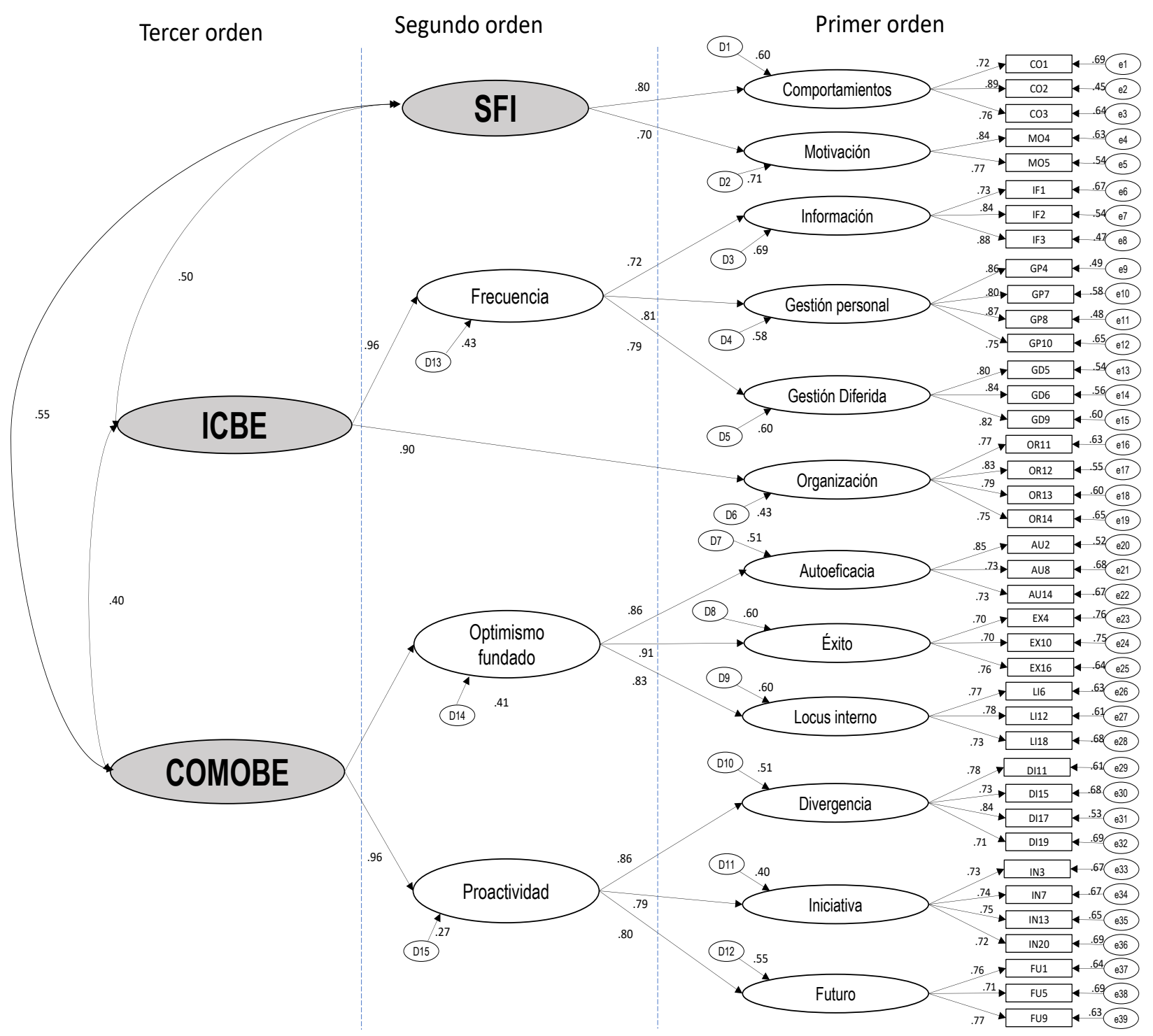

Fuente: elaboración propia.

En la Tabla 6, se comprueba que todas las dimensiones de segundo y tercer orden tienen un valor adecuado de fiabilidad compuesta $(\rho c)$, al ser superiores de .7, e inferiores a .95 (Bagozzi y Yi, 1988; Fornell y Larcker, 1981a; Hair et al., 2019), lo que evidencia la fiabilidad o consistencia interna de cada escala.

Además, ningún intervalo de confianza alcanza el valor mínimo de .7, tal y como sugiere la literatura (Hair et al., 2019). Las relaciones entre el factor de segundo orden y sus dimensiones son fuertes y significativas $(p<0.05)$. Respecto a la validez convergente, todas las dimensiones ofrecen un valor AVE superior al límite recomendable de .5 (Bentler, 2006; Hair et al., 2019). 


\section{Tabla 6}

Resultados de las dimensiones de orden superior

\begin{tabular}{|c|c|c|c|c|c|}
\hline Elementos & $\begin{array}{c}\text { Cargas } \\
(\lambda) \\
\text { CB- } \\
\text { SEM }\end{array}$ & $\begin{array}{c}\text { Cargas } \\
(\lambda) \\
\text { PLS- } \\
\text { SEM }\end{array}$ & $\begin{array}{c}\text { Intervalo } \\
\mathbf{R}^{\mathbf{2}}\end{array}$ & $\begin{array}{l}\text { Fiabilidad } \\
\text { comp. }\left(\rho_{c}\right) \\
{[5 \%-95 \%]}\end{array}$ & $\begin{array}{c}\text { AVE } \\
{[5 \%-95 \%]}\end{array}$ \\
\hline \multicolumn{6}{|c|}{ Medidas de segundo orden } \\
\hline \multicolumn{6}{|c|}{ Sistema Facilitador de inserción (SFI) } \\
\hline Comportamientos & .83 & .87 & $.52-.69$ & .872 & .578 \\
\hline Motivación & .70 & .83 & $.76-.79$ & {$[.850-.890]$} & {$[.532-.620$} \\
\hline \multicolumn{6}{|c|}{ Inventario de conductas de búsqueda de empleo (ICBE) } \\
\hline \multicolumn{6}{|l|}{ Frecuencia } \\
\hline Información & .72 & .77 & $.52-.59$ & .897 & .650 \\
\hline Gestión personal & .81 & .85 & $.66-.72$ & {$[.883-.809]$} & {$[.614-.687]$} \\
\hline Gestión diferida & .79 & .78 & $.61-.62$ & & \\
\hline \multicolumn{6}{|c|}{ Conductas motivadas de búsqueda de empleo (COMOBE) } \\
\hline \multicolumn{6}{|l|}{ Optimismo fundado } \\
\hline Autoeficacia & .88 & .83 & $.69-.77$ & .872 & .694 \\
\hline Éxito & .71 & .82 & $.50-.67$ & {$[.846-.895]$} & {$[.647-.739]$} \\
\hline Locus interno & .81 & .84 & $.66-.71$ & & \\
\hline \multicolumn{6}{|l|}{ Proactividad } \\
\hline Divergencia & .84 & .85 & $.71-.72$ & .880 & .711 \\
\hline Iniciativa & .87 & .85 & $.72-.76$ & {$[.855-.903]$} & {$[.663-.755$} \\
\hline Futuro & .83 & .82 & $.67-.69$ & & \\
\hline \multicolumn{6}{|c|}{ Medidas de tercer orden } \\
\hline \multicolumn{6}{|c|}{ Inventario de conductas de búsqueda de empleo (ICBE) } \\
\hline Frecuencia & .91 & .92 & $.83-.85$ & .903 & .824 \\
\hline Organización & .90 & .90 & $.81-.81$ & {$[.884-.920]$} & {$[.793-.852$} \\
\hline \multicolumn{6}{|c|}{ Conductas motivadas de búsqueda de empleo (COMOBE) } \\
\hline Optimismo fundado & .91 & .95 & $.83-.90$ & .910 & .629 \\
\hline Proactividad & .98 & .95 & $.90-.96$ & {$[.896-.923]$} & {$[.590-.667]$} \\
\hline
\end{tabular}

Fuente: Elaboración propia

Bondad de ajuste de los modelos de orden superior

Se siguen las recomendaciones de Franke y Sarstedt (2019) para estimar y validar modelos de orden superior en PLS-SEM y, al comparar los modelos de medida en cada una de las escalas con sus respectivos modelos de medida no correlacionados, ofrecen modelos de ajuste significativamente peores $(p<.05)$. Se aprecia para cada una de las escalas (SFI, ICBE y COMOBE) que el modelo de orden superior no es significativamente peor que el de orden inferior (Hair et al. 2010), tanto al comparar el segundo orden con el primer orden como al comparar el tercer orden con el segundo orden. Esto evidencia la validez y correcta estimación de los modelos de orden superior. Respecto a la validez discriminante de orden superior, en todas las construcciones, la raíz cuadrada del AVE es mayor que la correlación entre ellas (Fornell y 
Larcker, 1981a). Sobre la base de los resultados obtenidos se puede concluir que los constructos de orden superior son adecuados.

\section{Análisis de los resultados predictivos de los modelos de orden superior}

Con el fin de comprobar que las tres escalas miden aspectos diferentes, se comprueban los indicadores de multicolinealidad de determinación (R2) (Hair et al., 2019) y de valoración de los efectos (f2) (Cohen, 1992). Atendiendo a los resultados y a los intervalos de confianza, el poder predictivo entre COMOBE y SFI y entre ICBE y SFI son moderadas-altas, tanto en R2 como en f2. No así, la relación entre ICBE y COMOBE, que atendiendo a los intervalos de confianza son moderadas-débiles, tanto para R2 como para f2. Los tres constructos (SFI, ICBE, COMOBE) aunque relacionados con mayor o menor poder predictivo, puede considerarse que miden distintos aspectos.

\section{Conclusiones y Discusión}

El presente trabajo propone modificaciones en tres de las escalas que aplica el Servicio de Empleo en la Región de Murcia y realiza una aplicación piloto de las escalas mejoradas para conocer su capacidad para constituir una medida válida y fiable que pueda ser utilizada por técnicos en orientación e investigadores en este campo. Al construir escalas desde la especificidad de la situación de búsqueda de empleo se consiguen instrumentos más precisos y útiles que si se parte de medidas diseñadas desde enfoques más generalistas o de otros ámbitos de estudio como el clínico o sociológico (Beck et al., 1987; Buheji, 2019; Llosa Fernández, 2017). Los resultados confirman la validez del modelo propuesto que revela una estructura factorial sólida y ajustada, mejorada con los nuevos elementos.

La nueva configuración del SFI, más centrada en su concepción original, se posiciona como una referencia válida y fiable para valorar, con muy pocos ítems, los cuatro elementos del sistema: planificación, habilidades, información y motivación. Una opción interesante es utilizar el SFI en un primer contacto con las personas que inician la búsqueda de empleo, con el fin de poder ofrecer servicios complementarios como orientación, formación o actuaciones de apoyo, de manera preventiva. Aplicar escalas como el SFI, de sencilla y rápida respuesta, puede representar un importante ahorro en tiempo y recursos, sobre todo si se hace con grandes grupos de personas o a través de herramientas electrónicas que no precisan de la intervención directa de especialistas mediante entrevista individual.

EI ICBE, profundiza en los aspectos conductuales y mide de forma rigurosa tanto la variedad como la intensidad de las acciones de búsqueda de empleo, así como la capacidad organizativa y planificadora. Los nuevos elementos ayudan a distinguir en qué aspectos deben centrarse las intervenciones, según donde se muestre más competente la persona desempleada.

El COMOBE confirma también la validez de los nuevos ítems referidos a la conducta proactiva de búsqueda de empleo como un integrante confiable del modelo que, junto a las expectativas de autoeficacia, éxito y locus de control interno, constituyen una visión más completa de los aspectos motivacionales asociados a la búsqueda de empleo. 
Gracias al análisis factorial confirmatorio, se identifican con precisión componentes específicos muy relevantes en el proceso de búsqueda de empleo. Esto permite plantear en el futuro proyectos de investigación que respondan a cuestiones de relevancia en la definición de políticas activas de empleo y seleccionar las mejores técnicas o estrategias de intervención en orientación profesional. Se puede estudiar de forma independiente qué aspectos son determinantes en el proceso de desarrollo profesional y averiguar para un determinado grupo de personas, por ejemplo, si una autoeficacia baja retrasa su incorporación al trabajo o si la actitud proactiva de divergencia favorece el éxito en la búsqueda. También se pueden utilizar con seguridad los componentes de orden superior y valorar hasta qué punto una intervención orientadora modifica el optimismo fundado o si después de realizar una acción formativa aumenta nuestra proactividad de futuro, por ejemplo.

Las implicaciones de este trabajo suponen la puesta a disposición para los profesionales de la orientación de tres herramientas capaces de evaluar de forma fiable elementos importantes para prever la capacidad de las personas que desean pasar a la vida activa, sus fortalezas y recursos actitudinales para conseguir un empleo. Con la aplicación de las escalas, también se consigue mejorar el intercambio de información y conocimientos entre profesionales de la orientación e instituciones académicas y laborales, al poder realizar, con un lenguaje común, más y mejores estudios relevantes en el ámbito científico y diseñar programas de intervención desde una perspectiva más integradora entre el mundo educativo y el ámbito laboral.

\section{Limitaciones y nuevas líneas de actuación}

Una limitación de este estudio es la falta de referentes similares en otros servicios de empleo que pudieran dar lugar a estudios comparados. Tampoco pueden considerarse los aspectos valorados por estas escalas como exhaustivos y deben completarse con otros instrumentos como la evaluación de las competencias para la empleabilidad (Juárez Arall y Marqués Molías, 2019; Martínez Clares et al., 2018) o la resiliencia frente al desempleo (Beck, 2016; García León et al., 2018), por ejemplo. Una primera aplicación de las escalas mejoradas es sin duda alentadora por su amplia y clara estructura y su buen comportamiento estadístico, pero insuficiente para dimensionar su capacidad de evaluación y predicción en el campo de la orientación profesional.

Su mayor virtud es que nace de un esfuerzo colaborativo entre el ámbito académico y el servicio público de empleo. Desde la Universidad de Murcia se han diseñado las mejoras de las escalas y realizado los análisis de los datos y desde el Servicio de Empleo y Formación de la Región de Murcia se han proporcionado los datos y recursos necesarios para aplicar las escalas a personas usuarias de orientación profesional. Compartir datos, metodología e instrumentos entre servicios de empleo, universidades y otras entidades, configura un recurso que debemos conservar y desarrollar en el futuro como mejor camino en la construcción de soluciones eficaces al complejo y doloroso problema del desempleo.

Las futuras aplicaciones de las escalas en diferentes contextos y su utilización como medida objetiva del cambio en la motivación y la conducta de las personas desempleadas, representa una herramienta útil a la hora de valorar el impacto de las acciones de orientación y ayudará en la toma de decisiones para el diseño y selección de posibles modelos de intervención. 


\section{Referencias bibliográficas}

Agulló, E. (1998). La centralidad del trabajo en el proceso de construcción de la identidad de los jóvenes: una aproximación psicosocial. Psicothema, 10(1), 153-165. https://www.redalyc.org/pdf/727/72710114.pdf

Anderson, J. C., y Gerbing, D. W. (1988). Structural equation modeling in practice: A review and recommended two-step approach. Psychological Bulletin, 103(3), 411-423. https://psycnet.apa.org/buy/1989-14190-001

Aramburu, L. (2003). Enfoques cognitivo y conductual: evaluación de dos programas de intervención con desempleados. Psicothema, 15(2), 183-189.

http://www.psicothema.com/psicothema.asp?id=1043

Azrin, N. H., Flores, T. y Kaplan, S. J. (1975). Job-finding club: A group-assisted program for obtaining employment. Behaviour Research and Therapy, 13(1), 17-27. https://doi.org/10.1016/0005-7967(75)90048-0

Bagozzi, R. P. y Yi, Y. (1988). On the evaluation of structural equation models. Journal of the Academy of Marketing Science, 16(1), 74-94. https://doi.org/10.1007/BF02723327

Bagozzi, R. P. y Yi, Y. (2012). Specification, evaluation, and interpretation of structural equation models. Journal of the Academy of Marketing Science, 40(1), 8-34. https://doi.org/10.1007/s11747-011-0278-x

Beck, A. T., Brown, G., Steer, R. A., Eidelson, J. I. y Riskind, J. H. (1987). Differentiating anxiety and depression: A test of the cognitive content-specificity hypothesis. Journal of Abnormal Psychology, 96(3), 179-183. https://doi.org/10.1037/0021-843X.96.3.179

Beck, G. A. (2016). Surviving Involuntary Unemployment Together: The Role of Resilience-Promoting Communication in Familial and Committed Relationships. Journal of Family Communication, 16(4), 369-385. https://doi.org/10.1080/15267431.2016.1215315

Bentler, P. M. (2006). EQS 6 Structural equations modeling program manual. Encino, CA. http://www.econ.upf.edu/ satorra/CourseSEMVienna2010/EQSManual.pdf

Blanch, J. M. (2006). El trabajo como valor en las sociedades humanas. En A. Garrido, Coord., Sociopsicología del trabajo (57-98), UOC.

Buheji, M. (2019). Youth Unemployment Mitigation Labs-An Empathetic Approach for Complex Socio-Economic Problem. American Journal of Economics, 9(3), 93-105. https://doi.org/10.5923/i.economics.20190903.02

Caliendo, M., Cobb-Clark, D. A. y Uhlendorff, A. (2015). Locus of Control and Job Search Strategies. Review of Economics and Statistics, 97(1), 88-103. https://doi.org/10.1162/REST a 00459

Chou, C. P., Bentler, P. M. y Satorra, A. (1991). Scaled test statistics and robust standard errors for non-normal data in covariance structure analysis: a Monte Carlo study. The British Journal of Mathematical and Statistical Psychology, 44(2), 347-57.

http://www.ncbi.nlm.nih.gov/pubmed/1772802

Cifre, E., Vera, M. Sánchez-Cardona, I., y de Cuyper, N. (2018). Sex, Gender Identity, and Perceived Employability Among Spanish Employed and Unemployed Youngsters. Frontiers in Psychology, 9, 2467. https://doi.org/10.3389/fpsyg.2018.02467

Cohen, J. (1992). Statistical Power Analysis. Current Directions in Psychological Science, 1(3), 98- 


\section{1. https://doi.org/10.1111/1467-8721.ep10768783}

Consejo de la Unión Europea. (2008). Incluir mejor la orientación permanente en las estrategias permanentes de educación y formación permanente. Diario Oficial de la Unión Europea (2008/C 319/02). http://publications.europa.eu/resource/cellar/fa7d5979-b8f8-4688-8554ed9ff6d1d8cf.0007.02/DOC 1

Contador, I., Fernández-Calvo, B., Palenzuela, D. L., Miguéis, S. y Ramos, F. (2012). Prediction of burden in family caregivers of patients with dementia: A perspective of optimism based on generalized expectancies of control. Aging \& Mental Health, 16(6), 675-682. https://doi.org/10.1080/13607863.2012.684666

Fornell, C. y Larcker, D. F. (1981a). Evaluating Structural Equation Models with Unobservable Variables and Measurement Error. Journal of Marketing Research, 18(1), 39-50. https://doi.org/10.2307/3151312

Fornell, C. y Larcker, D. F. (1981b). Structural Equation Models with Unobservable Variables and Measurement Error: Algebra and Statistics. Journal of Marketing Research, 18(3), 382. https://doi.org/10.2307/3150980

Franke, G. y Sarstedt, M. (2019). Heuristics versus statistics in discriminant validity testing: a comparison of four procedures. Internet Research, 29(3), 430-447. https://doi.org/10.1108/IntR-12-2017-0515

García León, M. Á., González-Gomez, A., Robles-Ortega, H., Padilla, J. L. y Peralta-Ramirez, I. (2018). Propiedades psicométricas de la Escala de Resiliencia de Connor y Davidson (CDRISC) en población española. Anales de Psicología, 35(1), 33-40. https://doi.org/10.6018/analesps.35.1.314111

Gaskin, C. J. y Happell, B. (2014). On exploratory factor analysis: A review of recent evidence, an assessment of current practice, and recommendations for future use. International Journal of Nursing Studies, 51(3), 511-521. https://doi.org/10.1016/J.IJNURSTU.2013.10.005

Gold, A. H., Malhotra, A. y Segars, A. H. (2001). Knowledge Management: An Organizational Capabilities Perspective. Journal of Management Information Systems, 18(1), 185-214. https://doi.org/10.1080/07421222.2001.11045669

Hair, J. F., Black, W. C., Babin, B. J. y Anderson, R. E. (2010). Multivariate Data Analysis. (Pearson, Ed.). https://www.pearson.com/us/higher-education/program/Hair-Multivariate-Data-Analysis7th-Edition/PGM263675.html

Hair, J. F., Ringle, C. M. y Sarstedt, M. (2013). Partial Least Squares Structural Equation Modeling: Rigorous Applications, Better Results and Higher Acceptance. Long Range Planning, 46((12)), 1-12. https://doi.org/10.1016/.Irp.2013.08.016

Hair Jr., J. F., M. Hult, G. T., Ringle, C. M., Sarstedt, M., Castillo, J., Cepeda, G. A. y Roldán, J. L. (2019). Manual de Partial Least Squares Structural Equation Modeling PLS-SEM. (OmniaScience, Ed.).

Hefetz, A. y Liberman, G. (2017). El análisis factorial exploratorio: una guía breve con ejemplos. Cultura y Educación, 29(3), 542-562. https://dialnet.unirioja.es/servlet/articulo?codigo $=6264041$

Henseler, J., Ringle, C. M. y Sarstedt, M. (2015). A new criterion for assessing discriminant validity in variance-based structural equation modeling. Journal of the Academy of Marketing Science, 43(1), 115-135. https://doi.org/10.1007/s11747-014-0403-8 
Hernández-Fernaud, E., Ramos-Sapena, Y., Negrín, F., Ruiz-De La Rosa, C. I. y Hernández, B. (2011). Empleabilidad Percibida y Autoeficacia para la Búsqueda de Empleo en Universitarios Perception of Employability and Self-Efficacy for Job Seeking in University Students, 27, 2011-131. https://doi.org/10.5093/tr2011v27n2a5

INEM. (2000a). Desarrollo de los Aspectos Personales para la Ocupación (DAPO).

INEM. (2000b). Tutoría idividualizada (TI).

Juárez Arall, J. y Marqués Molías, L. (2019). Aspectos de la competencia digital para la empleabilidad//Digital competence aspects for employability. Revista Española de Orientación y Psicopedagogía, 30(2), 67. https://doi.org/10.5944/reop.vol.30.num.2.2019.25339

Karsten, P., Vastamäki, J. y Moser, K. (2016). Frustration of Life Goals Mediates the Negative Effect of Unemployment on Subjective Well-Being. Journal of Happiness Studies, 17(2), 447-462. https://doi.org/10.1007/s10902-014-9603-7

Koufteros, X., Babbar, S. y Kaighobadi, M. (2009). A paradigm for examining second-order factor models employing structural equation modeling. International Journal of Production Economics, 120(2), 633-652. https://doi.org/10.1016/J.IJPE.2009.04.010

Liu, S., Huang, J. L. y Wang, M. (2014). Effectiveness of job search interventions: a meta-analytic review. Psychological Bulletin, 140(4), 1009-41. https://doi.org/10.1037/a0035923

Llosa Fernández, J. A. (2017). Incertidumbre laboral y salud mental en trabajadores precarios y desempleados: un enfoque analítico e interventivo. http://digibuo.uniovi.es/dspace/handle/10651/45562

MacKenzie, S. B. y Podsakoff, P. M. (2012). Common Method Bias in Marketing: Causes, Mechanisms, and Procedural Remedies. Journal of Retailing, 88(4), 542-555. https://doi.org/10.1016/J.JRETAI.2012.08.001

Mardia, K. V. (1970). Measures of Multivariate Skewness and Kurtosis with Applications. Biometrika, 57(3), 519-530. https://doi.org/10.2307/2334770

Martínez Clares, P., González Lorente, C. y Rebollo Quintela, N. (2018). Competencias para la empleabilidad: un modelo de ecuaciones estructurales en la Facultad de Educación. Revista de Investigación Educativa, 37(1), 57-73. https://doi.org/10.6018/rie.37.1.343891

McNamee, S. y Gergen, K. J. (1996). La Terapia como construcción social. Paidós.

Montilla, S. (2003). Orientación profesional para el empleo: un esquema de trabajo multidimensional. Revista de psicología del trabajo y de las organizaciones, 19(1), 25-57. http://www.redalyc.org/articulo.oa?id=231318051002

Montilla, S. (2005). Diferencias individuales de los desempleados y constructos motivacionales: una relación de interdependencia predictora del desempleo estructural. Revista de psicología del trabajo y de las organizaciones, 21(3), 269-297. https://www.redalyc.org/articulo.oa?id=231317624005

Mortimer, J. T., Kim, M., Staff, J. y Vuolo, M. (2016). Unemployment, Parental Help, and Self-Efficacy During the Transition to Adulthood. Work and Occupations, 43(4), 434-465. https://doi.org/10.1177/0730888416656904

Nieto-Flores, M. P., Berrios, M. P. y Extremera, N. (2019). Autoeficacia de búsqueda como mediadora de la inteligencia emocional y la búsqueda activa de empleo. Revista de 
Psicología Social, 34(1), 86-109. https://doi.org/10.1080/02134748.2018.1537652

Nieto Flores, M. P., Berrios, P. y Extremera, N. (2015). Recursos personales asociados a diferentes indicadores de éxito en la búsqueda activa de empleo: una revisión sistemática. Behavioral Psychology / Psicología Conductual, 23(2), 373-392.

O'Hanlon, W. H. y Weiner-Davis, M. (1990). En busca de soluciones: un nuevo enfoque en psicoterapia. Paidos.

Palenzuela, D. L. (1989). Control personal: Un enfoque integrativo-multidimensional. In 2nd Convention of Portuguese Psychologists Association: International Conference. Lisboa, Portugal.

Pan, J., Guan, Y., Wu, J., Han, L., Zhu, F., Fu, X. y Yu, J. (2018). The interplay of proactive personality and internship quality in Chinese university graduates' job search success: The role of career adaptability. Journal of Vocational Behavior, 109, 14-26. https://doi.org/10.1016/J.JVB.2018.09.003

Pérez-Vacas, C. (2016). Inserción laboral de universitarios desde la perspectiva psicosocial. http://dehesa.unex.es/handle/10662/4040

Piqueras, R., Izquierdo, T. y Rodríguez, A. (2018). Conductas y actitudes que determinan la duración del desempleo: influencia en desempleados mayores de 45 años de la Región de Murcia, España. Revista Interamericana de Psicología Ocupacional, 37(2). https://doi.org/10.21772/ripo.v37n2a01

Piqueras, R. y Rodríguez, A. (1997). Orientación profesional centrada en las soluciones. Psychosocial Intervention, 6(3), 317-338. https://journals.copmadrid.org/pi/art/d77f00766fd3be3f2189c843a6af3fb2

Piqueras, R., Rodríguez, A. y Palenzuela, D. L. (2016). Validación de una escala de control personal: una medida específica de las expectativas de control percibido de búsqueda de empleo. Revista de Psicología del Trabajo y de las Organizaciones, 32(3), 153-161. https://doi.org/10.1016/..rpto.2016.06.001

Piqueras, R., Rodríguez, A. y Rueda, C. (2008). Expectativas y duración del desempleo. Revista de Psicología del Trabajo y de las Organizaciones, 24(2), 25-57. http://www.redalyc.org/articulo.oa?id=231316495001

Preuss, M. y Hennecke, J. (2018). Biased by success and failure: How unemployment shapes locus of control. Labour Economics, 53, 63-74. https://doi.org/10.1016/j.labeco.2018.05.007

Rego-Agraso, L. y Rial-Sánchez, A. (2018). ¿Por qué elegir formación profesional? Satisfacción, motivaciones y expectativas del alumnado sobre el empleo y la formación. Revista Española de Orientación y Psicopedagogía, 28(3), 43.

https://doi.org/10.5944/reop.vol.28.num.3.2017.21618

Rindskopf, D. y Rose, T. (1988). Some Theory and Applications of Confirmatory Second-Order Factor Analysis. Multivariate Behavioral Research, 23(1), 51-67. https://doi.org/10.1207/s15327906mbr2301 3

Saks, A. M., Zikic, J. y Koen, J. (2015). Job search self-efficacy: Reconceptualizing the construct and its measurement. Journal of Vocational Behavior, 86, 104-114. https://doi.org/10.1016/J.JVB.2014.11.007

Salessi, S. y Omar, A. (2017). Comportamientos proactivos en el trabajo: una puesta al día. Revista Argentina de Ciencias del Comportamiento, 9(3), 82-103. 
https://revistas.unc.edu.ar/index.php/racc/article/view/17800/Salessi Omar

Salessi, S. y Omar, A. (2018). Comportamientos proactivos en el trabajo: adaptación y análisis psicométrico de una escala. Actualidades en Psicología, 32(124), 33-49. https://doi.org/https://doi.org/10.15517/ap.v32i124.30642

SEF. (2007). Murcia Orienta. Murcia: Servicio Regional de Empleo y Formación - SEF- Región de Murcia.

Seibert, S. E., Crant, J. M. y Kraimer, M. L. (1999). Proactive personality and career success. Journal of Applied Psychology, 84(3), 416-427. https://doi.org/10.1037/0021-9010.84.3.416

SENAE. (2010). Orientación ocupacional individual. Santo Domingo: Servicio Nacional de Empleo (SENAE)- República Dominicana.

Sorić, P., Lolić, I., Claveria, O., Monte, E. y Torra, S. (2019). Unemployment expectations: A sociodemographic analysis of the effect of news. Labour Economics, 60, 64-74. https://doi.org/10.1016/j.labeco.2019.06.002

Sousa-Ribeiro, M., Sverke, M., Coimbra, J. L. y De Witte, H. (2018). Intentions to Participate in Training Among Older Unemployed People. Journal of Career Development, 45(3), 268-284. https://doi.org/10.1177/0894845316687669

Teré, F. (2019). Manual práctico de orientación profesional centrada en el empleo y la persona. Ediciones Eunate. https://eunateediciones.com/producto/manual-practico-de-orientacionprofesional-centrada-en-el-empleo-y-la-persona/

Tolentino, L. R., Garcia, P. R. J. M., Lu, V. N., Restubog, S. L. D., Bordia, P. y Plewa, C. (2014). Career adaptation: The relation of adaptability to goal orientation, proactive personality, and career optimism. Journal of Vocational Behavior, 84(1), 39-48. https://doi.org/10.1016/J.JVB.2013.11.004

Trice, A. D., Haire, J. R. y Elliott, K. A. (1989). A career locus of control scale for undergraduate students. Perceptual and Motor Skills, 69(2), 555-561.

Von Bertalanffy, L. (1993). Teoría general de los sistemas : fundamentos, desarrollo, aplicaciones. Fondo de Cultura Económica.

Watzlawick, P., Beavin, J. y Jackson, D. D. (1981). Teoría de la comunicación humana: Interacciones, patologías y paradojas. Herder.

West, S. G., Finch, J. F. y Curran, P. J. (1995). Structural equation models with nonnormal variables: Problems and remedies. In R. H. Hoyle (Ed.), Structural equation modeling: Concepts, issues, and applications (pp. 56-75). Sage, Inc. https://psycnet.apa.org/record/1995-97753-004 


\section{Apéndice}

\section{Estructura de las escalas mejoradas}

\section{SFI}

SFI/Comportamientos

CO1 Tengo toda la información que necesito para conseguir trabajo.

CO2 Me organizo habitualmente la búsqueda de empleo.

CO3 Domino las técnicas de búsqueda de empleo (currículum, anuncios, entrevistas, Internet, etc.).

SFI/Motivación

MO4 Me siento muy animada/o para buscar trabajo.

MO5 Creo que voy a conseguir pronto empleo.

ICBE

ICBE/FRECUENCIA/Información

IF1 Pregunto a familiares o conocidos sobre empleo.

IF2 Consulto anuncios de prensa.

IF3 Miro ofertas en tablones de anuncios.

ICBE/FRECUENCIA/Gestión personal

GP4 Visito oficinas de empleo o ETTs.

GP7 Acudo a las instituciones y organismos públicos.

GP8 Me presento a empresas privadas.

GP10 Consulto a expertos (orientadores, profesores, empresarios...).

ICBE/FRECUENCIA/Gestión diferida

GD5 Presento o envío mi currículum por escrito.

GD6 Utilizo webs de empleo y atiendo ofertas de internet.

GD9 Uso redes sociales (Facebook, Linkedin, etc.) para buscar trabajo.

ICBE/ORGANIZACIÓN

OR11 Utilizo una agenda para organizarme la búsqueda.

OR12 Me planifico la Búsqueda previamente.

OR13 Dedico unas horas determinadas del día a la búsqueda.

OR14 Marca las horas semanales que dedicas a buscar empleo.

\section{COMOBE}

COMOBE/OPTIMISMO FUNDADO/Autoeficacia

AU2 Soy capaz de soportar los inconvenientes que conlleva la búsqueda de empleo.

AU8 Estoy capacitada/o para superar los rechazos cuando busco empleo.

AU14 Creo que puedo comportarme adecuadamente en una entrevista de trabajo.

COMOBE/OPTIMISMO FUNDADO/Éxito

EX4 Creo que estaré poco tiempo en el paro.

EX10 Tengo muchas esperanzas de que me den trabajo.

EX16 Creo que hay muchas posibilidades de que termine encontrando un buen empleo.

COMOBE/OPTIMISMO FUNDADO/Locus de control interno 
LI6 Cuanto más me esfuerce en buscar trabajo más posibilidades tendré de encontrarlo.

LI12 Encontrar trabajo es algo que va a depender, fundamentalmente, de mí.

LI18 Encontrar trabajo dependerá del tiempo que dedique a buscarlo.

\section{COMOBE/PROACTIVIDAD/Divergencia}

DI11 Confío mucho en mi capacidad para mostrar mis habilidades en la selección de personal.

DI15 Busco trabajo en diferentes puestos o actividades laborales.

DI17 Reviso y modifico mi forma de buscar trabajo para conseguir mejores oportunidades.

DI19 Me imagino trabajando en distintos sitios y con diferentes responsabilidades.

COMOBE/PROACTIVIDAD/Iniciativa

IN3 Prefiero darme a conocer a las empresas antes que esperar una oferta.

IN7 Creo que hay que tomar la iniciativa en la búsqueda de empleo.

IN13 Me gusta demostrar que puedo tomar la iniciativa en mis responsabilidades laborales.

IN20 Me ofrezco a trabajar en empresas, aunque no estén buscando personal.

\section{COMOBE/PROACTIVIDAD/Futuro}

FU1 Ante una selección, procuro prever cuales de mis habilidades interesarán más a la empresa.

FU5 Soy capaz de adivinar qué esperan de mí los empresarios.

FU9 Me gusta imaginar cómo será el proceso de selección para prever posibles dificultades.

* Código de los ítems: 2 letras para la dimensión y el no de orden en el formulario de su escala

Fecha de entrada: 30 diciembre 2019

Fecha de revisión: 03 julio 2020

Fecha de aceptación: 23 septiembre 2020 